\title{
Splenocyte proliferation and anaphylaxis induced by BSA challenge in a D-galactose- induced aging mouse model
}

\author{
JI-HUN PARK ${ }^{1,2}$, TAE-SAENG CHOI \\ ${ }^{1}$ Department of Nanobiomedical Science and BK21 PLUS NBM Global Research Centre for Regenerative Medicine, Dankook University, \\ Chonan, Republic of Korea \\ ${ }^{2}$ Department of Microbiology, College of Medicine, Dankook University, Chonan, Republic of Korea
}

\begin{abstract}
We previously found a cross-reactive autoantibody that bound to bovine serum albumin generated in a D-galactose-induced aging mouse model. Also, we confirmed that other reducing sugars (glucose and fructose) could induce the formation of autoantibody, and only following subcutaneous injection, not oral or intraperitoneal administration. Mice that had never been exposed to bovine serum albumin produced an anti-bovine serum albumin autoantibody following repeated subcutaneous injection of $D$-galactose (D-gal). In this study, we investigated the involvement of the adaptive immune system in the production of this autoantibody. In particular, we examined bovine serum albumin-induced splenocyte proliferation and bovine serum albumin-induced active cutaneous and systemic anaphylaxis in D-gal-treated mice. We find our results particularly interesting: bovine serum albumin stimulates splenocyte proliferation and induces both active cutaneous and systemic anaphylaxis in D-gal-treated mice. In summary, our results suggest that adaptive immune response participates in the autoantibody formation against bovine serum albumin in D-gal-treated mice.
\end{abstract}

Key words: autoantibody, D-galactose-induced aging model mice, splenocyte, anaphylaxis.

(Cent Eur J Immunol 2016; (3): 324-327)

\section{Introduction}

The D-galactose-induced aging mouse model was produced by consecutive subcutaneous D-galactose (D-gal) injection over approximately six weeks [1]. This animal model shows an accelerated aging phenotype that may be due to excessive formation of reactive oxygen species (ROS) and the accumulation of advanced glycation end products (AGEs) [2-4]. We previously found that the cross-reactive autoantibody generated in this D-gal-induced aging mouse model did not bind to mouse serum albumin (MSA), but did bind to bovine serum albumin (BSA) [5]. Additionally, we found that administration of other reducing sugars (glucose or fructose) by subcutaneous injection also led to the formation of an autoantibody that cross-reacted with BSA [6]. In this study, we investigated the involvement of the adaptive immune system in the production of this autoantibody. BSA-induced splenocyte proliferation and BSA-induced active cutaneous and systemic anaphylaxis were examined in D-gal-treated mice.

\section{Material and methods}

\section{Animals and D-galactose treatments}

ICR female mice aged 6-8 weeks were used. The mice were provided drinking water and a normal diet ad libitum and kept under a 12-h light-dark cycle at $24 \pm 1^{\circ} \mathrm{C}$ and $50 \%$ humidity. Mice were randomly assigned into two experimental groups, each containing five mice. After a oneweek adaptation period, mice from each group were injected subcutaneously daily with $1000 \mathrm{mg} / \mathrm{kg}$ D-galactose (Sigma-Aldrich, St. Louis, MO, USA) or vehicle (0.9\% saline) as a control for approximately six weeks. Production of the anti-BSA antibody in D-gal-treated mice was confirmed by enzyme-linked immunosorbent assay (ELISA) [6]. After six weeks splenocytes and serum were prepared from D-gal-treated and saline-treated mice. All animal studies were conducted in compliance with the Guidelines for the Care and Use of Research Animals established by Dankook University Animal Studies Committee.

Correspondence: Tae-Saeng Choi, Room 403, Department of Microbiology, College of Medicine, Dankook University, 119 Dandae-ro, Dongnam-gu, Cheonan 330-714, Republic of Korea, e-mail: tschoi@ dankook.ac.kr Submitted: 22.10.2015; Accepted: 01.12.2015 


\section{Measurement of splenocyte proliferation}

Mice were killed by cervical dislocation, and spleens were aseptically removed and stripped of fat. Single-cell suspensions were obtained by grinding the spleens with a syringe plunger against a fine steel mesh [7]. Erythrocytes were lysed with ammonium chloride haemolysis buffer $\left(0.8 \% \mathrm{NH}_{4} \mathrm{Cl}\right.$ with $0.1 \mathrm{mM}$ EDTA) and then washed twice in complete RPMI-1640 Medium. Splenocytes $\left(5 \times 10^{5}\right.$ cells/well $)$ were plated in triplicate in 96-well culture plates and cultured in RPMI-1640 Medium supplemented with $5 \%$ foetal bovine serum (FBS) at $37^{\circ} \mathrm{C}$ in a humidified $5 \% \mathrm{CO}_{2}$ incubator. Cells were stimulated with various concentrations of BSA or MSA. Cells were also stimulated with lipopolysaccharides (LPS) at $10 \mu \mathrm{g} /$ well as a positive control. After 48 hours of culture, WST-1 reagent (Cayman, MI, USA) was added, cells were incubated for 2.5 hours, and viability was assessed by measuring the optical density (OD) at $450 \mathrm{~nm}$. The obtained values were normalised by subtraction of media or stimulator (BSA or MSA) baselines without cells.

\section{Evaluation of active cutaneous and systemic anaphylaxis}

For active cutaneous anaphylaxis, D-gal-treated mice were challenged by intradermal injection of $10 \mu \mathrm{g}$ BSA in $20 \mu \mathrm{l}$ saline (left) or saline only (right) into the shaved back. The mice were immediately injected with $100 \mu \mathrm{l}$ of $1 \%$ Evan's blue via the tail vein. Mice were sacrificed after 20 minutes and the back skin removed from the site of injection (approximately $1 \mathrm{~cm} \times 1 \mathrm{~cm}$ ). Evan's blue was extracted with $500 \mu \mathrm{l}$ formamide (Sigma-Aldrich, St. Louis, MO, USA) at $70^{\circ} \mathrm{C}$ for three hours. OD was measured at $620 \mathrm{~nm}$. For systemic anaphylaxis, D-gal-treated mice were injected intravenously (in the tail vein) with the indicated concentrations of BSA in $100 \mu \mathrm{l}$ saline and observed for one hour after the challenge. Symptoms were evaluated according to the following scoring system [8]: 0, no symptoms; 1 , scratching and rubbing around the nose and head; 2 , puffiness around the eyes and mouth, diarrhoea, pilar erecti, reduced activity, and/or decreased activity with increased respiratory rate; 3 , wheezing, laboured respiration, and cyanosis around the mouth and tail; 4, no activity after prodding or tremor and convulsion; and 5, death. Dexamethasone (Dexa, $50 \mu \mathrm{g} / 100 \mu \mathrm{l}$ in saline) was injected intraperitoneally in D-gal-treated mice 2, 24, and 48 hours before BSA challenge [9].

\section{Measurement of plasma histamine levels}

To determine plasma histamine levels, blood was collected 30 minutes after intravenous BSA challenge. For blood plasma preparation, whole blood was obtained by incising the tail vein with a sharp surgical blade and then centrifuged at $1500 \mathrm{x} g$ for 15 minutes at room temperature. Histamine was assayed on serum samples using an enzyme immunoassay kit (Labor Diagnostika Nord LND, Germany) as described by the manufacturer.

\section{Statistical analyses}

Statistical differences were subjected to one-way analysis of variance (ANOVA). Data are shown as the mean \pm standard error of the mean (SEM), and significance was defined as $p<0.05$ or 0.01 .

\section{Results}

\section{Splenocyte proliferation from D-galactose- treated mice is induced by $\mathrm{BSA}$}

The induction of splenocyte proliferation by BSA in D-gal-treated mice is shown in Fig. 1. As a positive control, LPS-stimulated splenocyte proliferation was increased to a comparable level in both control and D-gal-treated mice. The addition of BSA induced splenocyte proliferation in D-gal-treated mice in a concentration-dependent manner but not in control mice $(p<0.01)$. There was no change in proliferation in either group (control or D-gal-treated) by the addition of MSA.

Splenocytes were treated with different concentrations of BSA or MSA for 48 hours. Cells cultured in medium only served as a negative control. The positive control group was cultured with LPS alone. Splenocyte proliferation was measured by the WST-1 method as described in the text. Data values are expressed as the mean \pm S.E.M. from five mice per group. Significant differences from the media group were designated as $p<0.01$.

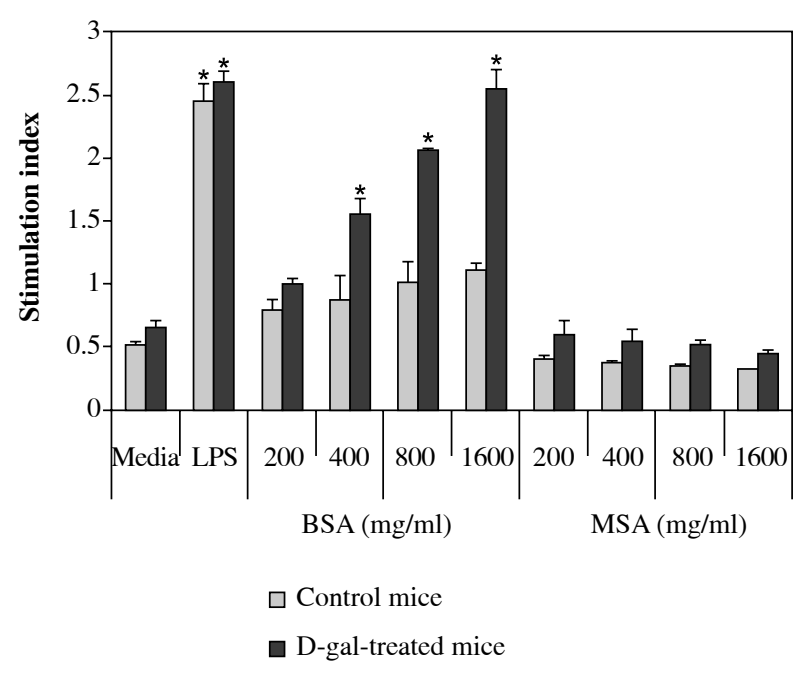

Fig. 1. Effect of bovine serum albumin and mouse serum albumin on the in vitro proliferation of mouse splenocytes from D-galactose-treated mice 


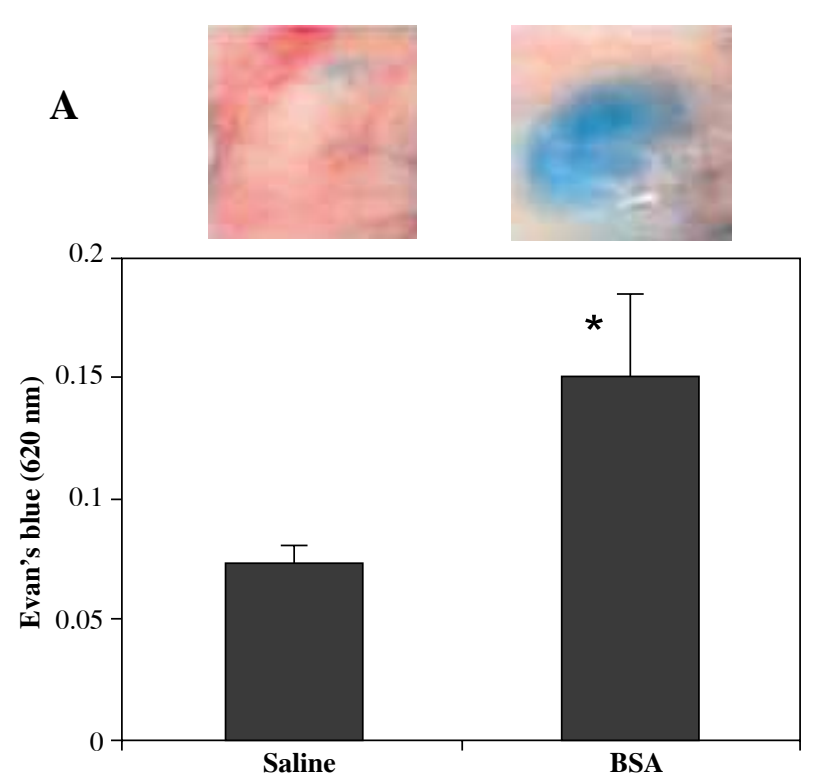

$$
\text { C }
$$

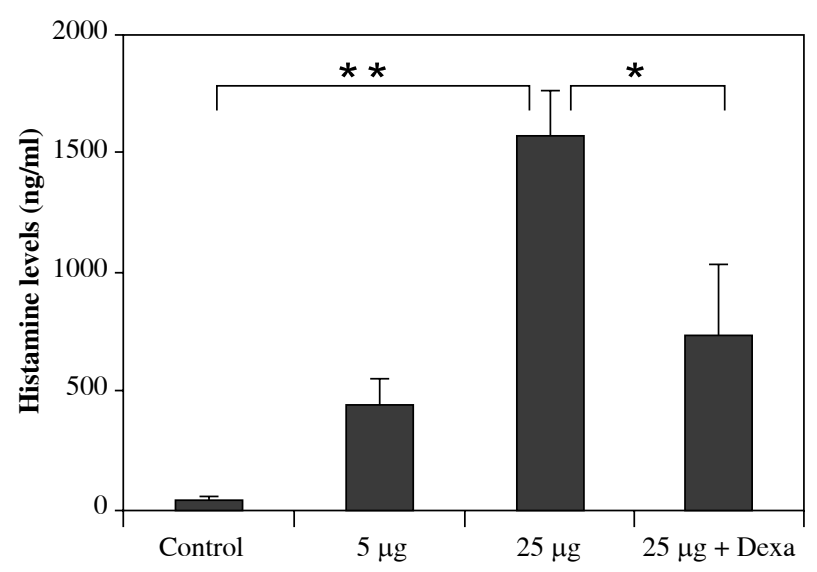

Induction of active cutaneous and systemic anaphylaxis by BSA challenge in D-galactosetreated mice

An active cutaneous anaphylaxis test was performed by intradermal injection of BSA into the back skin of control and D-gal-treated mice. As shown in Fig. 2A, extravasation of Evan's blue dye in D-gal-treated mice was significantly higher compared with control mice $(p<0.05)$.

For a systemic anaphylaxis test, mice were injected intravenously with 5,10 , or $25 \mu \mathrm{g}$ BSA. One group of mice was also pre-treated with Dexa before BSA challenge. Mice were observed and evaluated for one hour according to a scoring system for anaphylaxis (see "Material and methods"). The results indicate that the severity of anaphylaxis is dependent on the concentration of BSA. Also, the anaphylactic symptoms induced by BSA chal-

\section{B}

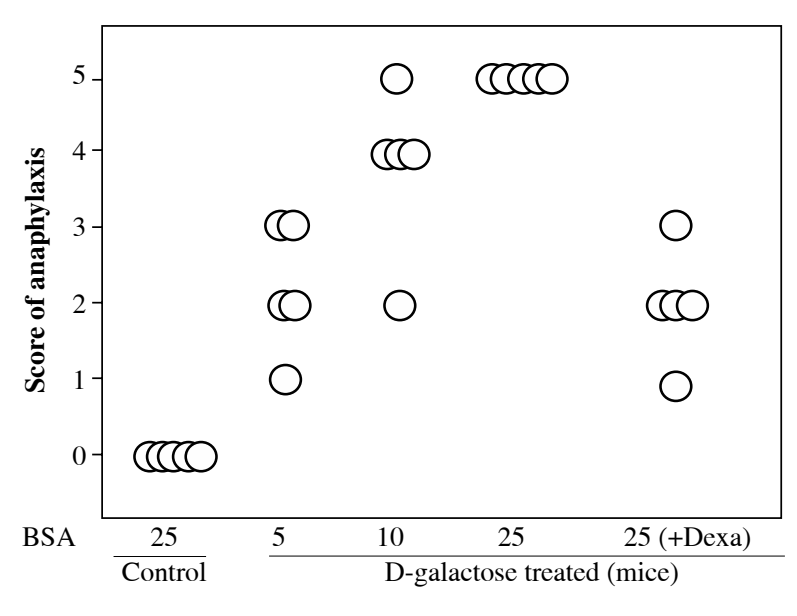

Fig. 2. BSA induced cutaneous and systemic anaphylaxis. A) D-galactose-treated mice were actively sensitised with $10 \mu \mathrm{g}$ bovine serum albumin (BSA) or saline. Representative example of leakage of Evan's blue dye injected and extracted from the back skin and read at $620 \mathrm{~nm}$ using a microplate reader. B) D-galactose-treated mice were challenged intravenously with BSA $(5,10,25 \mu \mathrm{g} /$ mouse $)$ or BSA plus dexamethasone. The symptoms of anaphylaxis were observed for one hour and scored as described in "Material and methods". Circles indicate individual mice. C) Plasma samples were collected from D-galactose-treated mice at 30 minutes after BSA challenge. Histamine levels were determined by ELISA. Data values are expressed as the mean \pm S.E.M. from five mice per group. Significant differences were designated as $* p<0.05$ or $* * p<0.01$

lenge were partially relieved with pre-treatment of Dexa in D-gal-treated mice (Fig. 2B). Next, we measured the levels of histamine, a plasma marker of systemic anaphylaxis, at 30 minutes after BSA challenge. BSA challenge did not increase plasma histamine levels in control mice, but did increase histamine levels more than $10(5 \mu \mathrm{g})$ or $50(25 \mu \mathrm{g})$ times control levels in D-gal-treated mice (Fig. 2C). On the other hand, pre-treatment with Dexa inhibited the increase in histamine levels by BSA challenge following D-gal treatment.

\section{Discussion}

We recently reported on the formation of cross-reactive autoantibodies binding to BSA, but not MSA, in mice subcutaneously injected repeatedly with reducing sugars [5]. Additionally, we confirmed that the formation of this auto- 
antibody is dependent on the route of administration (i.e., not oral or intraperitoneal but only subcutaneous) [6]. It is interesting to note that mice that had never been exposed to BSA produced an anti-BSA autoantibody, but only with repeated subcutaneous injections of D-gal. Our first question, however, was whether the adaptive immune system is involved in the production of this autoantibody. To address this, we performed an in vitro splenocyte proliferation assay in the presence of BSA and in vivo cutaneous and systemic anaphylaxis assays with BSA as an antigen. Our results showed that the proliferation of splenocytes prepared from D-gal-treated mice was induced by the addition of BSA but not MSA. This result may reflect the fact that the autoantibody cross-reacts with BSA but not with MSA. In other words, we confirmed the existence of lymphocytes that perceived BSA as an antigen in D-gal-treated mice.

Administration of BSA induced cutaneous and systemic anaphylaxis in D-gal-treated mice. In general, anaphylaxis, barring exceptional cases such as exercise, sunlight, etc., is caused by re-exposure to the previous sensitised antigen. In this regard, D-gal-treated mice were probably sensitised by some unknown endogenous protein(s) that share(s) an epitope with BSA. In previous studies, we confirmed that the IgG antibody cross-reacts with BSA [5, 6]. Many studies have shown that mouse anaphylaxis is mediated by $\operatorname{IgE}$, which stimulates histamine and platelet activating factor (PAF), or by IgG, which stimulates PAF [10-12]. In regard to systemic anaphylaxis, we showed that plasma levels of histamine were increased in D-gal-treated mice. This result suggests the possibility of $\mathrm{IgE}$ production against BSA in D-gal-treated mice. Future studies will be required to determine the different isotypes of antibodies produced in D-gal-treated mice.

This study was supported by research funds provided by Dankook University in 2014.

The authors declare no conflict of interest.

\section{References}

1. Song X, Bao M, Li D, et al. (1996): Advanced glycation in D-galactose induced mouse aging model. Mech Ageing Dev 108: 239-251.

2. Rojas A, Mercadal E, Fiqueroa H, et al. (2008): Advanced glycation and ROS: a link between diabetes and heart failure. Curr Vasc Pharmacol 6: 44-51.

3. Cui X, Zuo P, Zhang Q, et al. (2006): Chronic systemic D-galactose exposure induces memory loss, neurodegeneration, and oxidative damage in mice: protective effects of R-alphalipoic acid. J Neurosci Res 84: 647-654.

4. Park JH, Choi TS (2012): Polycystic ovary syndrome (PCOS)-like phenotypes in the D-galactose-induced aging mouse model. Biochem Biophys Res Commun 427: 701-704.

5. Park JH, Choi TS (2014): Production of cross-reactive autoantibody binding to bovine serum albumin in the D-galactose-induced aging mouse model. Am J Immunol 10: 3-9.
6. Park JH, Choi TS (2015): The production of cross-reactive autoantibodies that bind to bovine serum albumin in mice administered reducing sugars by subcutaneous injection. Cent Eur J Immunol 40: 25-29.

7. Cui L, Sun Y, Xu H, et al. (2013): A polysaccharide from Agaricus blazei Murill as a potent Th1 immunity-stimulating adjuvant. Oncol Lett 6: 1039-1044.

8. Li XM, Schofield BH, Huang CK, et al. (1999): A murine model of IgE-mediated cow`s milk hypersensitivity. J Allergy Clin Immunol 103: 206-214.

9. Overbergh L, Decallonne B, Branisteanu DD, et al. (2003): Acute shock induced by antigen vaccination in NOD mice. Diabetes 52: 335-341.

10. Strait RT, Morris SC, Yang M, et al. (2002): Pathways of anaphylaxis in the mouse. J Allergy Clin Immunol 109: 658-668.

11. Finkelman FD, Rothenberg ME, Brandt EB, et al. (2005): Molecular mechanisms of anaphylaxis: lessons from studies with murine models. J Allergy Clin Immunol 115: 449-458.

12. Khodoun MV, Strait R, Armstrong L, et al. (2011): Identification of markers that distinguish IgE- from IgG-mediated anaphylaxis. Proc Natl Acad Sci USA 108: 12413-12418. 